\title{
La tumeur odontogène adénomatoïde : Présentation d'un
}

\section{cas.}

\section{Campana F1, Lan R², Ordioni U33, Albertini AF4, Catherine $\mathrm{JH}^{2}$}

1. Centre Massilien de la Face - Exercice Privé - Marseille

2. Service d'Odontologie - Assistance publique - Hôpitaux de Marseille (AP-HM)

3. Centre Massilien de la Face - Exercice Privé - Marseille

4. Medipath - Exercice Privé - France

L'objectif de ce travail est de rapporter le cas d'une tumeur odontogène adénomatoïde (TOA) responsable d'une inclusion dentaire chez un enfant. Un enfant de 12 ans était adressé pour prise en charge chirurgicale de l'inclusion de 22. En dehors d'une amygdalectomie, il ne présentait aucun antécédent médical. L'examen clinique exo-buccal était sans anomalie. L'examen endo-buccal retrouvait la présence sur l'arcade de 62 et 63. L'imagerie par tomographie volumique à faisceau conique montrait la retenue de 22 par une lésion ostéolytique homogène, arrondie, de $7 \mathrm{~mm}$ de diamètre. L'exérèse chirurgicale associée à une prise en charge orthodontique était réalisée. L'histologie retrouvait une lésion encapsulée correspondant à une prolifération de cellules ovalaires formant de petites glandes régulières, sans mitose. Le stroma tantôt lâche, tantôt hyalin, comprenait des microcalcifications. L'étude immunohistochimique était positive pour AE1-AE3 et CK14, et négative pour la Ps100 et CK5/6. Après relecture de confirmation, le diagnostic de TOA était retenu.

La TOA a été décrite la première fois en 1909 par James. Elle est classée par l'OMS comme une tumeur odontogène et représente 3 à $7 \%$ de celles-ci (1). Elle peut survenir à tout âge de la vie, mais plus de la moitié des cas surviennent à l'adolescence, avec une prédominance féminine (sexe ratio F:M 1,9:1) $(1,2,3)$. La population asiatique semble plus atteinte (2). Sa localisation est quasi essentiellement intra-osseuse et préférentiellement au maxillaire $(1,2,3)$. La TOA est souvent associée avec une dent permanente retenue, en général une canine (3). On décrit ainsi 3 formes : péri-coronaire (75\% des cas), extra-coronaire (20\%) et périphérique $(5 \%)(2,3)$. Radiologiquement, la lésion est ostéolytique, le plus souvent uniloculaire, ronde, mimant un kyste dentigère lorsqu'elle est associée à une dent. Des opacités sont retrouvées dans $77 \%$ des cas (3). L'aspect histologique est varié, le plus typique étant celui d'une prolifération nodulaire de cellules épithéliales cuboïdes ou en colonnes. Parfois, comme dans notre cas, les cellules peuvent être tubulaires prenant un faux aspect de canal sécréteur. Du matériel calcifié peut être retrouvé (1). Le traitement est chirurgical et les récidives sont rares.

f.campana@centremassiliendelaface.com

\section{Bibliographie}

1- Barnes L et al. Pathology and genetics of head and neck tumours. Lyon: IARC Press; 2005.

2- Ide F, Mishima K, Kikuchi K, Horie N, Yamachika S, Satomura K, Shimoyama T, Sakashita H, Saito I, Kusama K. Development and growth of adenomatoid odontogenic tumor related to formation and eruption of teeth. Head Neck Pathol. 2011; 5:123-32.

3- Becker T, Buchner A, Kaffe I. Critical evaluation of the radiological and clinical features of adenomatoid odontogenic tumour. Dentomaxillofac Radiol. 2012; 41:533-40.

(C) The authors, published by EDP Sciences. This is an Open Access article distributed under the terms of the Creative Commons Attribution License 4.0 (http://creativecommons.org/licenses/by/4.0/) 